\title{
Supporting Information: Selective Plasmonic Enhancement of Electric- and Magnetic-Dipole Radiations of Er Ions
}

\author{
B. Choi, M. Iwanaga,* Y. Sugimoto, K. Sakoda, and H. T. Miyazaki \\ National Institute for Materials Science, 1-1 Namiki, Tsukuba 305-0044, Japan \\ *E-mail: iwanaga.masanobu@nims.go.jp
}

\section{S1 Numerical calculations}

We used the rigorously coupled-wave approximation (RCWA) method [1] to numerically calculate for reflection spectra of the nanocavity array. The RCWA method incorporates a scattering matrix algorithm [2] and can handle structures of stacked layers. The numerical precision for optical quantities such as reflectance was estimated to be less than $0.5 \%$ in the one-dimensionally periodic systems. The permittivity of gold was taken from the literature [3], and that of $\mathrm{SiO}_{2}$ and air was set to 2.1316 and 1.00054, respectively. EM field distributions were output by using a transfer-matrix method consistent with the RCWA method.

\section{S2 Fabrication procedure of plasmonic nanocavity array}

(a) $\mathrm{Er}^{3+}: \mathrm{SiO}_{2}$ deposition

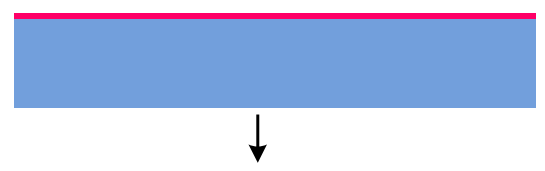

(b) $\mathrm{SiO}_{2}$ step deposition \& Annealing

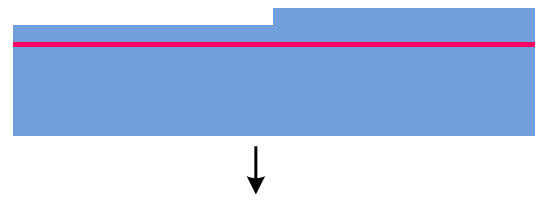

(c) Mask deposition \& Resist patterning

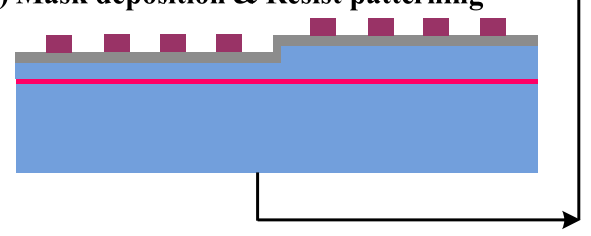

(d) Mask etching

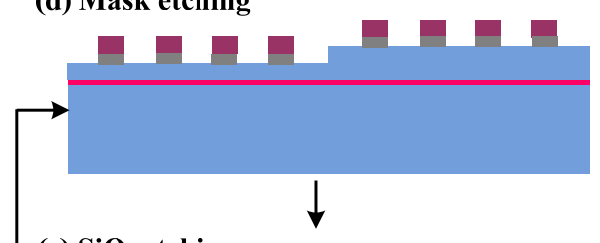

(e) $\mathrm{SiO}_{2}$ etching

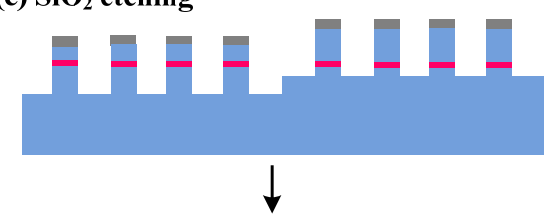

(f) Mask removal \& Au deposition

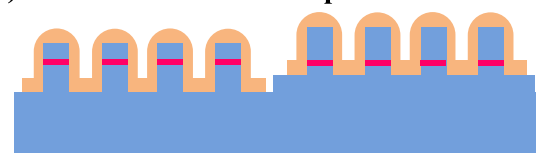

Figure S1: Schematics of fabrication procedure for Er-doped plasmonic nanocavity array. The depth of Er-doped layers were controlled in a deep subwavelength precision determined by the film-deposition precision.

Figure S1 illustrates the flowchart of the fabrication procedure of the plasmonic nanocavity array. The procedure has the following steps.

(a) At first, an Er-doped $\mathrm{SiO}_{2}$ film with a thickness of $20 \mathrm{~nm}$ was deposited by co-sputtering $\mathrm{Er}_{2} \mathrm{O}_{3}$ and $\mathrm{SiO}_{2}$ on quartz substrates of $0.5 \mathrm{~mm}$ thickness. We here note that bulk $\mathrm{Er}: \mathrm{SiO}_{2}$ for reference was independently deposited on quartz substrates. 
(b) Next, deposition of pure $\mathrm{SiO}_{2}$ was repeated with shifting a mask to form domains of different $\mathrm{SiO}_{2}$ thicknesses. Thus, the Er ions were embedded at different depths from the surface in the same substrate. Thermal annealing was then carried out in $\mathrm{O}_{2}$ atmosphere at $900{ }^{\circ} \mathrm{C}$ for 1 hour to activate Er ions. [4]

(c) After a 20-nm-thick Cr mask was deposited, electron-beam lithography with a negative resist was performed to make patterns for the nanocavity arrays. Various patterned area were produced in the range from $30 \times 30$ to $300 \times 300 \mu \mathrm{m}^{2}$, and width $W$ was produced from 70 to $130 \mathrm{~nm}$. To ensure the focusing of the electron beam on each domain with different $\mathrm{SiO}_{2}$ thickness, an automatic refocusing step was conducted before drawing in a new domain.

(d) After the development of the negative resist, inductively coupled plasma etching by $\mathrm{Cl}_{2}$ and $\mathrm{O}_{2}$ gases was performed to etch the Cr mask.

(e) Reactive ion etching by $\mathrm{CHF}_{3}$ gas was done to form the narrow $\mathrm{SiO}_{2}$ walls with the designed height.

(f) Single-layer Au film was deposited by $300 \mathrm{~nm}$ thickness with monitoring an indicator, to obtain a Au layer sufficiently thicker than the skin depth $(\sim 20 \mathrm{~nm})$.

In addition to the cavity arrays, two types of reference areas without nanopatterns were prepared in each step-like domain: one is a $\mathrm{SiO}_{2}$-overcoating area, which was made by additional deposition on the $\mathrm{Er}_{\mathrm{SiO}} \mathrm{Slayer}_{2}$ $\left(\mathrm{SiO}_{2} / \mathrm{Er}_{\mathrm{SiO}} / \mathrm{SiO}_{2}\right.$ structure), and another is an area on which $\mathrm{Au}$ was additionally deposited $\left(\mathrm{Au} / \mathrm{SiO}_{2} /\right.$

$\mathrm{Er}: \mathrm{SiO}_{2} / \mathrm{SiO}_{2}$ structure). These areas are used to measure the fundamental PL properties in $\mathrm{Er}: \mathrm{SiO}_{2}$. The latter was used as mirror configuration, as addressed in section S3. After going through the nanofabrication, the surface was sealed with epoxy resin and a quartz cover glass for protecting nanostructures and easy handling. Optical characterization of the nanocavity arrays was conducted from the substrate side.

In one process, the nanocavity arrays of Er-doped layers (red) of different depth were simultaneously fabricated in a quartz substrate. The key step was additional $\mathrm{SiO}_{2}$ deposition in Figure $\mathrm{S} 1 \mathrm{~b}$, where the depth position of the Er-doped layer was adjusted. We set the Er-doped layers at the designed positions within $1 \mathrm{~nm}$ precision. In experiment, incident light was shed from the bottom side in Figure S1.

\section{S3 Spectral Decomposition of ED and MD radiations}
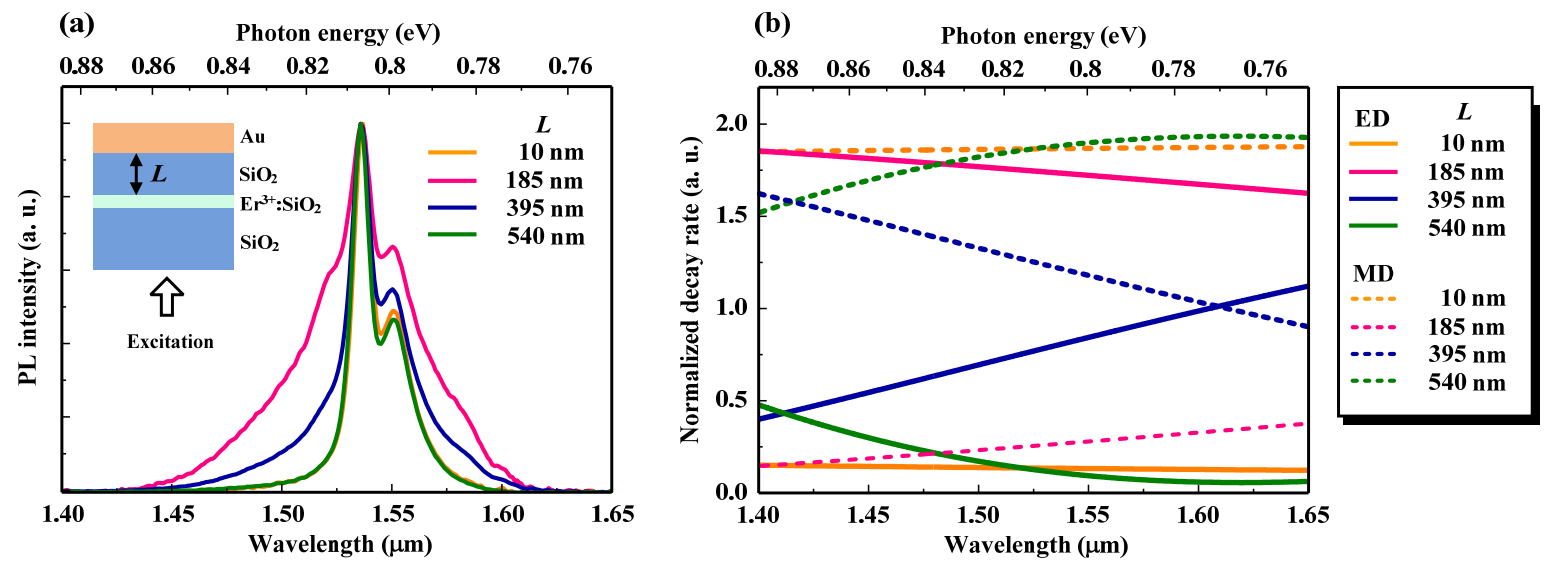

Figure S2: (a) PL spectra from the Er-embedded layers at several distances $L$ from the Au mirror. The inset draws the mirror configuration. (b) Normalized decay rates for four mirror configurations of Er-doped layers. ED-transition rates are shown with solid lines, and MD-transition rates with dashed lines.

As was shown in Figure 4a, the ratio of ED and MD radiations in the PL band at $1.5 \mu \mathrm{m}$ was determined through the analysis of mirror configuration that was reported in 1978 [5]. We here describe the decomposition procedure. 
Figure S2a shows measured PL spectra of Er ions at the four different distances from the Au mirror. The excitation wavelength was $0.523 \mu \mathrm{m}$. The spectra were normalized at the peak height. The inset depicts the configuration in the PL measurement. The Er ions were embedded in $\mathrm{SiO}_{2}$ matrix, and the Er-doped layers were set to be $20 \mathrm{~nm}$ thickness. The observed PL spectra depend on the distance $L$ from the Au mirror.

In the mirror configuration, analytical expressions of decay rate of spontaneous emission were already reported [5,6], and we evaluated decay ratios of decay rates by use of the analytical equations. The ratios for ED and MD transitions were defined by $\Gamma_{\mathrm{ED}} / \Gamma_{\mathrm{SiO}_{2}}$ and $\Gamma_{\mathrm{MD}} / \Gamma_{\mathrm{SiO}_{2}}$, respectively, where $\Gamma_{\mathrm{SiO}_{2}}$ is the decay rate of $\mathrm{Er}$ ions in bulk $\mathrm{SiO}_{2}$ matrix. We call the ratios normalized decay rates from now on. Figure $\mathrm{S} 2 \mathrm{~b}$ shows the normalized decay rates for the four positions of Er-doped layers.

Our aim here is to extract ED and MD components in the PL band of bulk $\mathrm{Er}_{\mathrm{SiO}}$. Let us write the PL intensity of bulk Er:SiO 2 as $I_{\mathrm{PL}}$. The $I_{\mathrm{PL}}$ consists of the ED and MD components $I_{\mathrm{ED}}$ and $I_{\mathrm{MD}}$, satisfying with

$$
I_{\mathrm{PL}}(\lambda)=I_{\mathrm{ED}}(\lambda)+I_{\mathrm{MD}}(\lambda)
$$

where $\lambda$ denotes observed wavelength. The $I_{\mathrm{ED}}(\lambda)$ and $I_{\mathrm{MD}}(\lambda)$ are solutions we are here seeking for.

In the mirror configurations in Figure S2a, we express the normalized PL intensities in the four configurations as $I_{\mathrm{PL}}^{(i)}(i=1,2,3,4)$, and assume the following relation:

$$
I_{\mathrm{PL}}^{(i)}(\lambda)=c_{i}\left[e^{(i)}(\lambda) I_{\mathrm{ED}}(\lambda)+m^{(i)}(\lambda) I_{\mathrm{MD}}(\lambda)\right]
$$

where $c_{i}$ is a normalization constant, and $e^{(i)}$ and $m^{(i)}$ are the normalized decay rates for ED and MD transitions, respectively, in Figure S2b. Equation (S2) for each $\lambda$ is written in a matrix form:

$$
\left(\begin{array}{l}
I_{\mathrm{PL}}^{(1)} \\
I_{\mathrm{PL}}^{(2)} \\
I_{\mathrm{PL}}^{(3)} \\
I_{\mathrm{PL}}^{(4)}
\end{array}\right)=\left(\begin{array}{ll}
c_{1} e^{(1)} & c_{1} m^{(1)} \\
c_{2} e^{(2)} & c_{2} m^{(2)} \\
c_{3} e^{(3)} & c_{3} m^{(3)} \\
c_{4} e^{(4)} & c_{4} m^{(4)}
\end{array}\right)\left(\begin{array}{l}
I_{\mathrm{ED}} \\
I_{\mathrm{MD}}
\end{array}\right) .
$$

The unknown factors $c_{i}$ were explored, as a generalized inverse matrix problem, under a constraint to satisfy Eqs. (S1) and (S3). We numerically found a set of solutions $\left\{c_{i}\right\}$ by using the least square method, and finally determined $I_{\mathrm{ED}}$ and $I_{\mathrm{MD}}$. Thus the decomposition of the PL spectrum in Figure 4a was implemented.

\section{References}

[1] Li, L. J. Opt. Soc. Am. A 1997, 14, 2758-2767.

[2] Li, L. J. Opt. Soc. Am. A 1996, 13, 1870-1876.

[3] Rakić, A. D.; Djurušić, A. B.; Elazar, J. M.; Majewski, M. L. Appl. Opt. 1998, 37, 5271-5283.

[4] Libertino, S.; Coffa, S.; Franzó, G.; Priolo, F. J. Appl. Phys. 1995, 78, 3867-3875.

[5] Chance, R. R.; Prock, A.; Silbey, R. Adv. Chem. Phys. 1978, 37, 1-65.

[6] Karaveli, S.; Zia, R. Opt. Lett. 2010, 35, 3318-3320. 